University of Wollongong

Research Online

Faculty of Engineering and Information

Faculty of Engineering and Information

Sciences - Papers: Part A

Sciences

$1-1-2015$

\title{
Valuation of options on oil futures under the 3/4 oil price model
}

Mohammed A. Aba Oud

Al Imam Mohammad Ibn Saud Islamic University, mamao481@uowmail.edu.au

Joanna Goard

University of Wollongong, joanna@uow.edu.au

Follow this and additional works at: https://ro.uow.edu.au/eispapers

Part of the Engineering Commons, and the Science and Technology Studies Commons

Research Online is the open access institutional repository for the University of Wollongong. For further information contact the UOW Library: research-pubs@uow.edu.au 


\title{
Valuation of options on oil futures under the $3 / 4$ oil price model
}

\author{
Abstract \\ Recently, an empirically-validated one-factor model with a 3/4-power diffusion term was introduced in the \\ literature to model oil prices and value futures contracts on oil. In this paper, we provide an exact and \\ analytic approximation for European call option prices on futures under a 3/4-power futures model. The \\ analytic approximation, valid for short times to expiry is then calibrated to market prices. Results from the \\ calibration show that the analytic approximation formula outperforms current popular options on futures \\ formulae in capturing market prices. \\ Disciplines \\ Engineering | Science and Technology Studies \\ Publication Details \\ Aba Oud, M. \& Goard, J. M. (2015). Valuation of options on oil futures under the 3/4 oil price model. \\ International Journal of Theoretical and Applied Finance, 18 (8), 1550050-1-1550050-12.
}




\title{
Valuation of Options on Oil Futures under the 3/4 Oil Price Model
}

\author{
MOHAMMED A. ABA OUD \\ Department of Mathematics and Statistics, Al Imam Mohammad Ibn Saud Islamic University \\ (IMSIU), Riyadh, Kingdom of Saudi Arabia \\ maabaoud@imamu.edu.sa \\ JOANNA GOARD \\ School of Mathematics and Applied Statistics, University of Wollongong, \\ Northfields Ave, Wollongong, 2522, NSW, Australia \\ joanna@uow.edu.au \\ Received (Day Month Year) \\ Revised (Day Month Year)
}

\begin{abstract}
Recently, an empirically-validated one factor model with a 3/4-power diffusion term was introduced in the literature to model oil prices and value futures contracts on oil. In this paper we provide an exact and an analytic approximation for European call option prices on futures under a 3/4-power futures model. The analytic approximation, valid for short times to expiry is then calibrated to market prices. Results from the calibration show that the analytic approximation formula outperforms current popular options on futures formulae in capturing market prices.
\end{abstract}

Keywords: options on futures; options on oil; commodity options.

\section{Introduction}

Oil is one of the most important of the world's commodities. In 2012, oil provided about $33.1 \%$ of the world's energy needs and in the future, oil is expected to continue to provide a leading component of the world's energy. With prices for crude oil often experiencing high levels of volatility, financial derivatives such as futures and options, have become increasingly important to financial institutions for the purposes of trading and risk management. The value of a financial derivative on oil typically depends upon the model that describes the movement of oil prices. In the paper by AbaOud \& Goard (2015), several one-factor diffusion models were investigated in their ability to describe the dynamics of oil prices. All of the models took the form $d P=A(P, t) d t+B(P, t) d Z$ where here and in the rest of the paper, $P\left(=P_{t}\right)$ denotes the price of oil at time $t, A(P, t)$ is the drift term, $B(P, t)$ the diffusion term and $d Z$ is an increment in a standard Wiener process with real probability measure $\mathbb{P}$. The investigation included the Geometric Brownian Motion (GBM) model used by Brennan \& Schwartz (1985), which assumes that $A(P, t)=\mu P$ and $B(P, t)=\sigma P$ where $\mu$ and $\sigma$ are constant, and the mean-reverting model used 
by Schwartz (1997) where $A(P, t)=\eta P(\mu-\ln (P)), B(P, t)=\sigma P$. It was found that the '3/4-model' $d P=(\hat{a} \sqrt{P}+\hat{b} P) d t+\hat{\sigma} P^{\frac{3}{4}} d Z$ with $\hat{b}<0$ performed best in explaining the behaviour of crude oil prices. We note that the $3 / 4$-model describes a mean-reverting process with a reversion rate dependent on the price level. This means that after a large spike, the price can potentially quickly decrease. As well, when $2 \hat{a}>\hat{\sigma}^{2}$, with $P_{0}>0$ then $P_{t}$ can never be negative. Further, futures prices under a risk-neutral model

$$
d P=(a \sqrt{P}+b P) d t+\sigma P^{\frac{3}{4}} d \tilde{Z}
$$

where the market price of risk is such that the real process has the same form as the risk-neutral process and where the Wiener process $Z$ is replaced by $\tilde{Z}$, another Wiener process under an equivalent risk-neutral probability measure $\mathbb{Q}$, performed best in fitting and forecasting futures prices on oil.

We note that as stated by Ribeiro \& Hodges (2005), reduced form models dominate the literature and practice on energy derivatives. They are especially attractive from the practitioners' perspective when they provide closed form solutions to evaluate financial derivatives, which in turn facilitate calibration and computational implementation of the models.

The aim of this paper is to provide valuations for European call options on futures when futures prices follow a 3/4 model, and compare their ability in capturing market prices with those prices formulated under the GBM and Schwartz models. In Section 2, we give the exact solution in the form of an integral for the price of European call option contracts on futures and then an analytic approximation formula valid for short times to expiry. We note that it is such options with short tenor which actually dominate the options markets. The results of empirical tests which compare the performance of our formula with the performance of prices under the GBM and Schwartz models, in their ability to describe market option prices, are presented in Section 3. As well in Section 3, we present the results of delta-hedging simulations and compare the three models' performances with respect to minimising hedging error. In Section 4 we present our conclusion.

\section{Options on Futures Valuation}

Assuming that the risk-neutral spot price of oil follows equation (1.1), then the futures price, $F$, has a zero drift in the risk-neutral world and its process can be approximated for short times to expiry by

$$
d F=\sigma F^{\frac{3}{4}} d \tilde{Z} .
$$

It can be shown then (see e.g Wilmott (1998)) that the partial differential equation (PDE) governing the price of a call option contract on a futures, $C(F, t)$, with strike price $K$ and expiry $T$ is

$$
\frac{\partial C}{\partial t}+\frac{\sigma^{2} F^{\frac{3}{2}}}{2} \frac{\partial^{2} C}{\partial F^{2}}-r C=0
$$


to be solved subject to the final condition

$$
C(F, T)=\max (F-K, 0)
$$

The exact solution to $(2.2 \mathrm{a}-\mathrm{b})$ can be found using the transition density function of $F$ which is given by

$$
f\left(F, t ; F_{T}, T\right)=\frac{4 \sqrt{F} I_{2}\left(\frac{16\left(F F_{T}\right)^{\frac{1}{4}}}{\sigma^{2}(T-t)}\right)}{\sigma^{2}(T-t) F_{T}} \exp \left\{-\frac{8\left(\sqrt{F}+\sqrt{F_{T}}\right)}{\sigma^{2}(T-t)}\right\}
$$

(see Goard (2006)) where $I_{\nu}($.$) is the modified Bessel function of the first kind$ of order $\nu$ (see e.g. Abramowitz \& Stegun (1965)). In this case the exact solution of $(2.2 \mathrm{a}-\mathrm{b})$ can be found as the present value of the expected payoff under the risk-neutral measure $\mathbb{Q}$ i.e.

$$
\begin{aligned}
C(F, t) & =e^{-r(T-t)} E_{t}^{\mathbb{Q}}\left(\max \left[F_{T}-K, 0\right]\right) \\
& =e^{-r(T-t)}\left[\int_{K}^{\infty}(y-K) f(F, t ; y, T) d y\right] .
\end{aligned}
$$

Equation (2.4) involves an integral which would normally need to be solved numerically. In the following result we provide a simple approximate solution to (2.2a-b) valid for short times to expiry and which only involves simple mathematical functions. This would facilitate pricing and calibration of options with short tenor.

Main Result: Given that the futures prices, $F$, follows the risk-neutral process (2.1), then an approximate solution for a European call option with exercise price $K$ and expiry $T$, written on the futures contract, valid for short times to expiry is given by

$$
\begin{aligned}
C(F, t) & =\frac{\sqrt{T-t}\left(4 K \theta_{1}+\theta_{2}(F-K)\right)}{4 \sqrt{\pi \theta_{1}}} \exp \left\{-\frac{(F-K)^{2}}{4 K^{2} \theta_{1}(T-t)}\right\} \\
& +\frac{F-K}{2} \operatorname{erfc}\left(-\frac{F-K}{2 K \sqrt{\theta_{1}(T-t)}}\right) \\
\text { where } \theta_{1} & =\frac{\sigma^{2}}{2 \sqrt{K}} \text { and } \theta_{2}=\frac{3 \sigma^{2}}{4 \sqrt{K}} .
\end{aligned}
$$

Proof. We let $\tau=T-t$ so equation $(2.2 \mathrm{a})$ can be written as

$$
\frac{\partial C}{\partial \tau}=\frac{\sigma^{2} F^{\frac{3}{2}}}{2} \frac{\partial^{2} C}{\partial F^{2}}-r C
$$

which needs to be solved subject to the initial condition $C(F, 0)=\max (F-K, 0)$ and the boundary conditions $C(0, \tau)=0$ and $C(F, \tau) \sim F-K$ as $F \rightarrow \infty$. For 
a small time to expiry $\tau$ we let $\tau=\varepsilon \grave{\tau}$ where $\varepsilon$ is a small parameter $0<\varepsilon \ll 1$, and assume that the solution can be written as a series in $\varepsilon$ i.e.

$$
C(F, \grave{\tau})=\sum_{i=0}^{\infty} \varepsilon^{i} C_{i}(F, \grave{\tau})
$$

Substituting $\tau=\varepsilon \grave{\tau}$ and (2.7) into (2.6), we get the first two terms of our series as

$$
C_{0}(F, \grave{\tau})+\varepsilon C_{1}(F, \grave{\tau})= \begin{cases}(F-K)(1-\varepsilon r \grave{\tau}) & F \geq K, \\ 0 & \text { otherwise . }\end{cases}
$$

From equation (2.8) it can be observed that the expansion is continuous, but not differentiable at $F=K$. This suggests a corner layer at $F=K$ where very fast changes occur in the derivative of the solution, but not in the value of the solution. Solution (2.8) is therefore invalid in this region and is thus termed our "outer" solution. We now analyse the solution in the inner region by introducing a stretching variable $x=\frac{F-K}{\sqrt{\varepsilon} K}$ and rescale $C(F, \grave{\tau})=K \sqrt{\varepsilon} W(F, \grave{\tau})$. The choice of power $\frac{1}{2}$ in $\sqrt{\varepsilon}$ is a well-balanced choice and ensures that the coefficient of the second-order derivative term is not small compared to the other coefficients. Equation (2.6) then becomes

$$
\frac{\partial W}{\partial \grave{\tau}}=\frac{\sigma^{2}}{2 \sqrt{K}}(1+\sqrt{\varepsilon} x)^{\frac{3}{2}} \frac{\partial^{2} W}{\partial x^{2}}-r \varepsilon W
$$

which needs to be solved subject to the initial condition $W(x, 0)=\max (x, 0)$ and $W(x, \grave{\tau}) \sim x$ as $x \rightarrow+\infty$ and $W(x, \grave{\tau}) \rightarrow 0$ as $x \rightarrow-\infty$.

Now we expand $W(x, \grave{\tau})$ in terms of $\sqrt{\varepsilon}$ i.e.

$$
W(x, \grave{\tau})=\sum_{i=0}^{\infty} \varepsilon^{\frac{i}{2}} W_{i}(x, \grave{\tau})
$$

and substitute (2.10) into (2.9). Collecting terms of $O(1)$ we thus get an equation for $W_{0}(x, \grave{\tau})$, namely

$$
\frac{\partial W_{0}}{\partial \grave{\tau}}=\theta_{1} \frac{\partial^{2} W_{0}}{\partial x^{2}}, \quad \text { where } \theta_{1}=\frac{\sigma^{2}}{2 \sqrt{K}},
$$

to be solved subject to the conditions $W_{0}(x, 0)=\max (x, 0), W_{0}(x, \grave{\tau}) \sim x$ as $x \rightarrow$ $+\infty$ and $W_{0}(x, \grave{\tau}) \rightarrow 0$ as $x \rightarrow-\infty$. Equation (2.11) has a similarity solution $W_{0}(x, \grave{\tau})=\sqrt{\grave{\tau}} \phi(z)$ where $z=\frac{x}{\sqrt{\grave{\tau}}}$ which upon substituting in (2.11) yields the reduced equation $2 \theta_{1} \phi^{\prime \prime}+z \phi^{\prime}-\phi=0$ to be solved subject to the boundary conditions $\phi(z) \sim z$ as $z \rightarrow+\infty$ and $\phi(z) \rightarrow 0$ as $z \rightarrow-\infty$.

The solution to the above problem is easily found to be $\phi(z)=\sqrt{\frac{\theta_{1}}{\pi}} \exp \left\{-\frac{z^{2}}{4 \theta_{1}}\right\}+$ $\frac{z}{2} \operatorname{erfc}\left(-\frac{z}{2 \sqrt{\theta_{1}}}\right)$.

Hence $W_{0}(x, \grave{\tau})$ is given by

$$
W_{0}(x, \grave{\tau})=\sqrt{\frac{\theta_{1} \grave{\tau}}{\pi}} \exp \left\{-\frac{x^{2}}{4 \theta_{1} \grave{\tau}}\right\}+\frac{x}{2} \operatorname{erfc}\left(-\frac{x}{2 \sqrt{\theta_{1} \grave{\tau}}}\right),
$$


where $\theta_{1}$ is given in (2.11). Now collecting terms of $O(\sqrt{\varepsilon})$ we get an equation for $W_{1}(x, \grave{\tau})$ namely

$$
\frac{\partial W_{1}}{\partial \grave{\tau}}=\theta_{1} \frac{\partial^{2} W_{1}}{\partial x^{2}}+\theta_{2} x \frac{\partial^{2} W_{0}}{\partial x^{2}} \text { where } \theta_{2}=\frac{3 \sigma^{2}}{4 \sqrt{\mathrm{K}}}
$$

and where $\theta_{1}$ is given in (2.11). Equation (2.13) needs to be solved subject to the conditions $W_{1}(x, 0)=0$ and $W_{1}(x, \grave{\tau}) \rightarrow 0$ as $x \rightarrow \pm \infty$. As $W_{0}(x, \grave{\tau})$ satisfies (2.11) we find the particular solution ${ }^{\mathrm{a}}$ of (2.13) satisfying the given conditions as

$$
W_{1}(x, \grave{\tau})=\frac{\theta_{2} x \sqrt{\grave{\tau}}}{4 \sqrt{\pi \theta_{1}}} \exp \left\{-\frac{x^{2}}{4 \theta_{1} \grave{\tau}}\right\} .
$$

From $(2.12),(2.14)$ and $(2.10)$ we get the two-term inner expansion

$$
W(x, \grave{\tau})=\sqrt{\frac{\theta_{1} \grave{\tau}}{\pi}} \exp \left\{-\frac{x^{2}}{4 \theta_{1} \grave{\tau}}\right\}+\frac{x}{2} \operatorname{erfc}\left(-\frac{x}{2 \sqrt{\theta_{1} \grave{\tau}}}\right) \sqrt{\varepsilon}\left[\frac{\theta_{2} x \sqrt{\grave{\tau}}}{4 \sqrt{\pi \theta_{1}}} \exp \left\{-\frac{x^{2}}{4 \theta_{1} \grave{\tau}}\right\}\right] .
$$

Equation (2.15) is valid in the inner region, while equation (2.8) is valid in the outer region. Now we can match the outer and inner expansions to get the uniform expansion which is uniformly valid in both outer and inner regions. The uniform expansion can be found by combining the outer and inner expansions and then subtracting the common part, i.e. 'outer+inner-common'. In our solution as $\varepsilon \rightarrow 0$ the outer expansion coincides with the common part, so the inner expansion can be used to approximate the price of call option contracts. Hence, we get the price of a call option contract by undoing the change of variables in (2.15) to get (2.5).

Formula (2.5) can be more useful for pricing European call options with small times to expiry as compared to (2.4) as it involves no integration. For this reason also it is more convenient for calibration purposes.

\subsection{Accuracy of the analytic approximation formula}

To measure the accuracy of our new analytic approximation formula (2.5) we obtained values using this formula and compared them with the corresponding values obtained with the exact formula, equation (2.4), using numerical integration ${ }^{\mathrm{b}}$. We considered $T-t=\frac{3}{12}, \frac{2}{12}, \frac{1}{12}$, and for each value of $T-t$ we chose typical values $F=100, r=0.01$, five values for the strike price $(K=98,99,100,101,102)$ and three values for the volatility $(\sigma=0.1,0.2,0.3)$. The signed percentage error (SPE):

$$
\mathrm{SPE}=\frac{C_{\text {approximate }}-C_{\text {exact }}}{C_{\text {exact }}} \times 100 \%
$$

${ }^{\mathrm{a}} \mathrm{We}$ use the following results: If $u_{y}=\frac{1}{2} u_{x x}$ and $v_{y}=\frac{1}{2} v_{x x}+u$, then $v=$ $y u$ is a particular solution.If $u_{y}=\frac{1}{2} u_{x x}$ and $v_{y}=\frac{1}{2} v_{x x}+x u$, then $v=x y u+$ $\frac{y^{2}}{2} u_{x}$ is a particular solution.

${ }^{2}$ The mathematics package Maple is used to obtain numerical integration values with relative error tolerance $1 \times 10^{-8}$. 
Table 1. Signed percentage error (SPE) and absolute error (AP) of analytic approximation formulae (2.5) and (2.4).

\begin{tabular}{|c|c|c|c|c|c|c|c|}
\hline \multirow{2}{*}{$\sigma$} & \multirow{2}{*}{$K$} & \multicolumn{2}{|c|}{$T-t=\frac{3}{12}$} & \multicolumn{2}{|c|}{$T-t=\frac{2}{12}$} & \multicolumn{2}{|c|}{$T-t=\frac{1}{12}$} \\
\hline & & $\operatorname{SPE}(\%)$ & $\mathrm{AE}\left(\times 10^{-2}\right)$ & $\operatorname{SPE}(\%)$ & $\mathrm{AE}\left(\times 10^{-2}\right)$ & SPE (\%) & $\operatorname{AE}\left(\times 10^{-2}\right)$ \\
\hline \multirow{5}{*}{0.1} & 98 & 0.25 & 0.52 & 0.17 & 0.34 & 0.08 & 0.17 \\
\hline & 99 & 0.25 & 0.31 & 0.17 & 0.19 & 0.08 & 0.09 \\
\hline & 100 & 0.25 & 0.16 & 0.17 & 0.09 & 0.08 & 0.03 \\
\hline & 101 & 0.25 & 0.06 & 0.17 & 0.03 & 0.08 & 0.01 \\
\hline & 102 & 0.25 & 0.02 & 0.15 & 0.01 & 0.01 & 0.00 \\
\hline \multirow{5}{*}{0.2} & 98 & 0.25 & 0.63 & 0.17 & 0.39 & 0.08 & 0.18 \\
\hline & 99 & 0.25 & 0.46 & 0.17 & 0.27 & 0.08 & 0.11 \\
\hline & 100 & 0.25 & 0.32 & 0.17 & 0.17 & 0.08 & 0.06 \\
\hline & 101 & 0.26 & 0.21 & 0.17 & 0.10 & 0.09 & 0.03 \\
\hline & 102 & 0.26 & 0.13 & 0.18 & 0.06 & 0.09 & 0.01 \\
\hline \multirow{5}{*}{0.3} & 98 & 0.26 & 0.78 & 0.17 & 0.47 & 0.08 & 0.20 \\
\hline & 99 & 0.26 & 0.62 & 0.17 & 0.36 & 0.09 & 0.14 \\
\hline & 100 & 0.26 & 0.49 & 0.17 & 0.27 & 0.09 & 0.09 \\
\hline & 101 & 0.26 & 0.38 & 0.18 & 0.19 & 0.09 & 0.06 \\
\hline & 102 & 0.27 & 0.29 & 0.18 & 0.14 & 0.09 & 0.04 \\
\hline \multicolumn{2}{|c|}{ average } & & 0.36 & & 0.20 & & 0.08 \\
\hline
\end{tabular}

and absolute error $(\mathrm{AE})$ for each couple $(K, \sigma)$ were calculated. Table 1 lists the results. From this table we note that the analytic approximation formula (2.5) slightly overprices option contracts compared with the exact solution. However for all the chosen $(\sigma, K)$ with $T-t=\frac{1}{12}$, the SPE values lied within a small range, namely $(0.01 \%, 0.09 \%)$. As time to expiry increased, the SPE values increased (as expected) but for all the chosen $(\sigma, K)$ with $T-t=\frac{3}{12}$ the SPE values remained very small and lay within $(0.25 \%, 0.27 \%)$. The average absolute errors were less than $0.08 \times 10^{-2}, 0.20 \times 10^{-2}$ and $0.36 \times 10^{-2}$ with $T-t=\frac{1}{12}, \frac{2}{12}, \frac{3}{12}$ respectively. This suggests that (2.5) provides an excellent approximation to the exact solution for times to expiry up to three months.

\subsection{Delta-Hedging}

As we have an explicit approximate formula for the European call option, we can differentiate it with respect to any of the variables and parameters to derive the 'Greeks'. For example the delta of the call option, i.e. the derivative of the call option value with respect to the futures price $F$ is given by

$$
\begin{aligned}
\Delta=\frac{\partial C(F, t)}{\partial F}= & \frac{2 \theta_{1} \theta_{2} K^{2} \sqrt{(T-t)}-\theta_{2}(F-K)^{2}}{8 \theta_{1} K^{2} \sqrt{\pi \theta_{1}(T-t)}} \exp \left\{-\frac{(F-K)^{2}}{4 K^{2} \theta_{1}(T-t)}\right\} \\
& +\frac{1}{2} \operatorname{erfc}\left(-\frac{F-K}{2 K \sqrt{\theta_{1}(T-t)}}\right) .
\end{aligned}
$$

In Section 3.2.3 we give the results of delta-hedging simulations. 


\section{Empirical Test}

In this section we examine the new analytic approximation formula (2.5) for European call option prices in its ability to capture market prices. In addition, we compare its performance with options prices corresponding to the GBM and Schwartz models. Under the assumption that the risk-neutral futures prices follow the GBM model with zero drift i.e. $d F=\sigma F d \tilde{Z}$, the price of a European call option contract is given by:

$$
\begin{aligned}
C(F, t) & =e^{-r(T-t)}\left[F N\left(d_{1}\right)-K N\left(d_{2}\right)\right] \\
\text { where } \quad d_{1} & =\frac{\ln \left(\frac{F}{K}\right)+\frac{\sigma^{2}}{2}(T-t)}{\sigma \sqrt{T-t}} \text { and } d_{2}=d_{1}-\sigma \sqrt{T-t} .
\end{aligned}
$$

(see Black \& Scholes (1973)), where $N($.$) is the cumulative distribution function$ of a standardised normal random variable. Its Delta is given by

$$
\Delta_{G B M}=\frac{\partial C(F, t)}{\partial F}=e^{-r(T-t)} N\left(d_{1}\right) .
$$

Under the assumption that the risk-neutral futures prices follow the Schwartz model with zero drift i.e. $d F=\sigma e^{-\eta(T-t)} F d \tilde{Z}$, the price of a European call option contract is given by:

$$
\begin{aligned}
& C(F, t)=e^{-r(T-t)}\left[F N\left(d_{3}\right)-K N\left(d_{4}\right)\right] \quad \text { where } \\
& d_{3}=\frac{\ln \left(\frac{F}{K}\right)+\frac{w^{2}}{2}(T-t)}{w \sqrt{T-t}}, \quad d_{4}=d_{3}-w \sqrt{T-t}, \quad w^{2}=\frac{\sigma^{2}}{2 \eta(T-t)}\left(1-e^{-2 \eta(T-t)}\right),
\end{aligned}
$$

(see Skorodumov (2008)) and its Delta is given by

$$
\Delta_{S c h}=\frac{\partial C(F, t)}{\partial F}=e^{-r(T-t)} N\left(d_{3}\right) .
$$

\subsection{Data description and estimation of parameters}

The options data used in our empirical test was selected from the New York Mercantile Exchange (NYMEX) and consisted of daily observations of European call option closing prices between the years 2009 and 2012, with the underlying asset being futures contracts for light sweet crude oil. We divided the relevant data into two groups: Group 1 with options of approximately one month (28-35 days) expiries and Group 2 with expiries of approximately two months (59 - 61 days). Table 2 presents the standard statistics for the options data used in our empirical test. The parameters in the models to be estimated were $\sigma$ for all models and $\eta$ for the Schwartz model. The estimated parameters (for each pricing formula and group) were chosen to provide the 'best' fit to our options data, and we chose to do this as follows:

We defined $H_{i}\left(\hat{H}_{i}\right)$ to be the market (estimated) price of the option contract $i$ and $e_{i}$ to be the error in contract $i$, i.e $e_{i}=\hat{H}_{i}-H_{i}$. Also for each pricing formula we let $\theta(j)$ be the parameter vector for group $j=1,2$. We used our data prices 
Table 2. Standard statistics for the options data.

\begin{tabular}{c|ccc|ccc}
\hline \hline \multirow{2}{*}{ statistics } & \multicolumn{3}{|c|}{ Group 1 (1410 observations) } & \multicolumn{3}{|c}{ Group 2 (1332 observations) } \\
& $F$ & $K$ & $T-t$ (in days) & $F$ & $K$ & $T-t$ (in days) \\
\hline \hline mean & 89 & 90.04 & 30.21 & 92.29 & 97.17 & 60.69 \\
standard deviation & 10.09 & 10.86 & 1.11 & 8.17 & 8.94 & 1.21 \\
\hline \hline
\end{tabular}

$\left(i=1, . ., N_{j}\right.$, where $N_{j}$ is the number of observations in group $j$ ) and minimised the sum of squares of errors (SSE) i.e. $\min S S E(\theta(j))=\sum_{i=1}^{N_{j}} e_{i}^{2}$. This resulted in the parameter vector $\theta(j)$ for each pricing formula and for each group $j=1,2$.

\subsection{Performance of options models}

\subsubsection{Comparison of Models}

For each group $j=1,2$ the following measures were used to compare errors in the performance of European call options on futures pricing models:

- The sum of squared errors: $S S E_{j}=\sum_{i=1}^{N_{j}}\left(\hat{H}_{i}-H_{i}\right)^{2}, \quad j=1,2$.

- The root mean squared errors: $R M S E_{j}=\sqrt{\frac{1}{N_{j}-q} \sum_{i=1}^{N_{j}}\left(\hat{H}_{i}-H_{i}\right)^{2}}, \quad j=1,2$,

where $q$ is the number of the parameters in the pricing formula.

Table 3. Estimated parameters, SSE and RMSE of option pricing models.

\begin{tabular}{c|ccc|ccc}
\hline \hline & \multicolumn{3}{|c|}{ Group 1 (1410 observations) } & \multicolumn{3}{c}{ Group 2 (1332 observations) } \\
Estimated & $\mathrm{Eq}(3.1)$ & $\mathrm{Eq}(3.2)$ & $\mathrm{Eq}(2.5)$ & $\mathrm{Eq}(3.1)$ & $\mathrm{Eq}(3.2)$ & $\mathrm{Eq}(2.5)$ \\
parameters & $(\mathrm{GBM})$ & $(\mathrm{Sch})$ & $(3 / 4$ model $)$ & $(\mathrm{GBM})$ & $(\mathrm{Sch})$ & $(3 / 4$ model $)$ \\
\hline \hline$\sigma$ & 0.31744 & 0.34909 & 0.97620 & 0.31441 & 0.34186 & 0.98253 \\
$\eta$ & & 2.37167 & & & 1.03612 & \\
\hline$S S E$ & 264.55501 & 264.39316 & 231.91119 & 438.57759 & 438.50930 & 415.25263 \\
$R M S E$ & 0.43331 & 0.43334 & 0.40570 & 0.57403 & 0.57398 & 0.55856 \\
\hline \hline
\end{tabular}

Table 3 lists the results of our analysis. In particular, we note that within the first group our new analytic approximation formula under the three-quarters model fits the option prices best and has the smallest SSE of approximately 231.91 and RMSE of $\$ 0.41$ per contract. The Schwartz model had the next smallest SSE of approximately 264.39 although the GBM model had the next smallest RMSE. Comparison of SSE and RMSE values within the second group (with 2 month maturities) agree with the results of the first group, but (as expected) errors increase with time to expiry. 
Table 4. Av. SPE and Av. UPE

\begin{tabular}{c|ccc|ccc}
\hline \hline \multirow{2}{*}{ M } & \multicolumn{3}{c|}{ Av. SPE } & \multicolumn{3}{c}{ Av. UPE } \\
& GBM & Sch & 3/4 model & GBM & Sch & 3/4 model \\
\hline \hline \multicolumn{7}{c}{ Group 1 } \\
\hline \hline ITM & -0.80 & -0.78 & 0.85 & 6.44 & 6.42 & 5.46 \\
ATM & 0.25 & 0.26 & 0.62 & 11.34 & 11.32 & 10.69 \\
OTM & 3.88 & 3.90 & 4.19 & 18.61 & 18.61 & 17.99 \\
\hline \hline \multicolumn{7}{c}{ Group 2 } \\
\hline \hline ITM & 4.46 & 4.47 & 6.64 & 5.90 & 5.91 & 6.97 \\
ATM & 4.38 & 4.40 & 5.64 & 7.33 & 7.34 & 7.22 \\
OTM & 2.84 & 2.85 & 3.13 & 17.68 & 17.68 & 17.54 \\
\hline \hline
\end{tabular}

In summary we can infer from our given data and empirical analysis, that the new analytic approximation formula for options prices (2.5) under the three-quarters model outperforms the other models in capturing short-tenor option prices.

\subsubsection{Examination of Market and Models Prices}

From the analysis above, the 3/4 model outperformed the GBM and Schwartz models in describing market prices. Next we examined more closely the differences between the market and model values.

For each model and group, we used the estimated parameters that best fit market prices (i.e. the parameters in Table 3) to compute model prices $\hat{C}_{i}$ for each strike price, to then compare with corresponding market prices $C_{i}$. For each option we calculated the corresponding signed percentage error i.e.

$$
S P E=\frac{\hat{C}_{i}-C_{i}}{C_{i}} \times 100 \%,
$$

unsigned percentage error i.e.

$$
U P E=\left|\frac{\hat{C}_{i}-C_{i}}{C_{i}}\right| \times 100 \%
$$

and moneyness $M$, defined by $M=\ln \left(\frac{F}{K}\right)$. Note that a positive (negative) $M$ value denotes that the option is in (out)-the-money. Results were grouped into the range of expiries (i.e. Group 1 and Group 2) and the average signed and unsigned percentage errors were calculated for the three groups of moneyness: options with $M \in[-0.05,0.05]$ were denoted by ATM (at-the-money), options with $M>0.05$ were denoted by ITM (in-the-money) and options with $M<-0.05$ were denoted by OTM (out-the-money). These are listed in Table 4. In particular, we make the following observations from the table:

A comparison of

- average SPE indicates that all model prices are on average above market prices for ATM and OTM options in both groups as well as for ITM options in Group 2 
(with expiries of two months). This suggests that market demand may have been quite low for such options.

- average UPE shows that the $3 / 4$ model achieved the lowest average UPE in all cases except for ITM options from Group 2, where the GBM and Schwartz models achieved lower errors.

Hence while we concluded in Section 3.2.1 that the 3/4 model performed best in fitting market prices of one and two month options, from a closer examination by grouping into moneyness categories, we see that the $3 / 4$ model performed best in fitting market values for all groups of moneyness and expiries tested, with the exception of ITM options with the larger expiry time of two months.

\subsubsection{Delta-Hedging Performance}

Delta-hedging is an important risk-management strategy used by many options traders. We now compare the performance of our 3/4 model with the GBM and Schwartz models in minimising hedging error whilst maintaining delta-hedged portfolios. Our data for this experiment consisted of 218 European call option contracts selected from the NYMEX. These contracts were equally divided into two groups (i.e. 109 contracts per group) where Group 1 options had expiries of one month and Group 2 options had expiries of two months. For each contract and model we assumed that the call option was written (i.e sold) and $\Delta=\frac{\partial C}{\partial F}$ futures contracts were held, with the hedge adjusted daily. Parameter values were chosen that best fit the model option price to the market price at the start of the simulation. The Delta for the 3/4 model is given in (2.17) and for the GBM and Schwartz models are given in Section 3. For each contract (and model) we compute their absolute hedging error at the option's expiry time as:

$$
H E= \begin{cases}\left|\bar{C} e^{r T}-K-X\right| & F_{T}>K, \\ \left|\bar{C} e^{r T}-X\right| & F_{T}<K,\end{cases}
$$

where $\bar{C}$ is the model call option value at the opening of the contract, $F_{T}$ is the futures price at the expiry of the option contract and $X$ is the cumulative cost of buying/selling the underlying futures.

Table 5 lists the results for the absolute hedging errors for the 218 contracts. From Table 5 we see that for both groups the lowest mean absolute hedging error was achieved by the $3 / 4$ model followed by the GBM. The standard deviation of the errors is also smallest with the $3 / 4$ model. Hence the $3 / 4$ model outperformed the other models in reducing the risks associated with delta-hedging the call options with expiries of one and two months.

We also note from Table 5 that all 3 models produce HE with similar distributional properties. For Group 1 the absolute hedging errors have relatively flat distributions while for Group 2, the distributions are more peaked. As expected, all the distributions have asymmetric tails extending towards more positive values. 
Table 5. Absolute hedging errors (standard statistics) .

\begin{tabular}{c|ccc|ccc}
\hline \hline statistics & \multicolumn{3}{|c|}{ Group 1 } & \multicolumn{3}{c}{ Group 2 } \\
& GBM & Sch & $3 / 4$ model & GBM & Sch & 3/4 model \\
\hline \hline mean & 0.54733 & 0.56400 & 0.52160 & 1.41598 & 1.47978 & 1.35852 \\
standard deviation & 0.46525 & 0.47252 & 0.42534 & 0.85548 & 0.90344 & 0.81954 \\
kurtosis & -0.15085 & -0.14357 & -0.17102 & 0.45729 & 0.09645 & 0.61572 \\
skewness & 0.80118 & 0.80674 & 0.79755 & 1.02115 & 0.84461 & 1.07962 \\
\hline \hline
\end{tabular}

\section{Conclusion}

Under the 3/4 risk-neutral model (2.1) for futures prices on oil, an exact solution and a simple approximate solution which only involves simple mathematical functions, are found for call options on oil futures. The approximate solution, which is valid for options with short tenor (a popular choice in the market) has been shown to outperform two current popular models, the GBM and Schwartz models, in capturing market prices for options with one and two month expiries. A closer inspection of the different models' performance by moneyness grouping showed that the $3 / 4$ model performed best in all moneyness categories with the exception of ITM options with two month expiries. Further endorsement for the $3 / 4$ model was provided by a delta-hedging experiment which showed that out of the three models tested, the minimum average absolute hedging error was achieved by the $3 / 4$ model. In conclusion the formulae for option prices under the $3 / 4$ model, namely (2.4) and the approximate solution for short-tenored options (2.5) could potentially provide very useful guides to traders.

\section{References}

M. A. Aba Oud \& J. Goard (2015) Stochastic Models for Oil Prices and the Pricing of Futures on Oil, Applied Mathematical Finance 22 (2), 189-206.

M. Abramowitz \& I. Stegun (1965) Handbook of Mathematical Functions With Formulas, Graphs, and Mathematical Tables. New York: Dover Publications.

F. Black \& M. Scholes (1973) The Pricing of Options and Corporate Liabilities, Journal of Political Economy $\mathbf{8 1}$ (3), 637-654.

M. Brennan \& E. Schwartz (1985) Evaluating Natural Resource Investments, Journal of Business 58, 135-157.

J. Goard (2006) Fundamental Solutions to Kolmogorov Equations via Reduction to Canonical Form, Advances in Decision Sciences 3, 1-24.

D.R. Ribeiro \& S.D. Hodges (2005) A Two-Factor Model for Commodity Prices and Futures Valuation, Journal of Futures Markets Nov, 1025-1044.

E. Schwartz (1997) The Stochastic Behaviour of Commodity Prices: Implications for Valuation and Hedging, Journal of Finance 3, 923-973.

B.B. Skorodumov (2008) Estimation of Mean Reversion in Oil and Gas Markets. Technical Report, MITSUI/2008-10-14. Available at skorodumov.net/pdf/report_oct_08.pdf

P. Wilmott (1998) Derivatives: The Theory and Practice of Financial Engineering. Chichester, England: John Wiley and Sons. 